\title{
Duty cycle variation methods for Bragg gratings: comparative study and optimal design
}

\author{
ANATOLE LuPU, ${ }^{1, *}$ \\ ${ }^{1}$ Centre de Nanosciences et de Nanotechnologies, CNRS, Université Paris-Saclay, C2N-10 Boulevard \\ Thomas Gobert-91120 Palaiseau cedex, France \\ *anatole.lupu@c2n.upsaclay.fr
}

\begin{abstract}
An analysis of spectral response apodization by duty cycle methods for gratingassisted optical filters is presented. The study is performed for the case of co-directional couplers, Bragg reflectors and mirrors. The modeling results show that the supersymmetric duty cycle (SDC) modulation method, initially considered for Parity-Time symmetric gratings [JSTQE 22(5), 35-41 (2016)], performs better than the other methods. An analytical approach to the transfer matrix of the modulated grating explains the better performance of the SDC method.
\end{abstract}

(C) 2021 Optical Society of America under the terms of the OSA Open Access Publishing Agreement

\section{Introduction}

Grating assisted devices are important elements for many applications in integrated optics [1]. Such devices are successfully modeled by a set of coupled mode differential equations. Filters employing Bragg scattering are of two types: the reflection type such as corrugated waveguide filters [2] and transmission type, such as the grating assisted directional couplers (GADC) [3]. If the coupling strength is constant, the spectral response of the filter is limited by the relatively large sidelobes level. The level of sidelobes can be reduced by tapering the coupling profile. One of the most efficient tapering techniques is the variation of the grating duty cycle. As this method is compatible with a binary etching process (constant etching depth), it is particularly interesting when Bragg gratings are realized by a surface corrugation technique, as it is often the case for photonic crystals or similar 1D or 2D periodic structures.

Several of duty cycle variations methods have been described in the literature [4-9]. The basic duty cycle (BDC) variation method suggested by Sakata [5], is using the coupling strength dependence on the grating duty cycle, i.e. the ratio of the grating ridge width $(\Lambda+\Delta) / 2$ to the grating period $\Lambda$, where $\Delta / 2$ represents the grating section length variation (Fig. 1a). The "Phase Shifted Pair Gratings" method (PSPG) [6], alternates on a double grating period length $(2 \Lambda)$ sections of length $(\Lambda+\Delta) / 2$ and $(\Lambda-\Delta) / 2$ separated by $\Lambda / 2$ intervals (Fig. 1c). The off-resonance method (ORM) [7] is based on alternating gratings periods of lengths $\Lambda+\Delta$ and $\Lambda-\Delta$ (Fig. 1b). The concatenated gratings method (CGM), suggested by Wiessman [8,9], seems similar to ORM, but grating periods are of length $\Delta$ and $\Lambda-\Delta$, as represented in Fig. 1d. A natural question arises: what type of duty cycle variation method is most effective? Is there a better method of duty cycle modulation?

The objective of this paper is to perform a comparative analysis of the effectiveness of different duty cycle variation methods. The focus is on the relationship between the spectral response behavior and the symmetry of duty cycle modulated gratings. This relationship is found by using a perturbation approach for the transfer matrix of the modulated grating. The developed approach provides the main guidelines for the optimal design of duty-cycle modulated gratings. 




Fig. 1. Schematic of grating duty cycle variation methods. a) Basic duty cycle variation method, b) Phase Shifted Pair Gratings method, c) Off-Resonance method, d) Concatenated gratings method.

\section{BDC modulated grating symmetry and apodized spectral response behavior}

To highlight the relationship between the symmetry of the duty cycle modulated grating and its spectral properties, we consider the example of a GADC with a rectangular shaped grating (Fig. 2). The grating is decomposed in sections of length $\Lambda_{\mathrm{i}+}$ and $\Lambda_{\mathrm{i}-\text { where the coupling }}$ coefficient is respectively positive: $\kappa_{\mathrm{i}+}$ and negative: $\kappa_{\mathrm{i}}$. The coupling coefficient $\kappa$ depends of the overlap of the two guided modes and the perturbation of index $\tilde{n}^{2}$ that the grating represents. It is proportional to the following integral: $\kappa \propto \int \tilde{n}^{2} E_{1} E_{2}^{*} \mathrm{dxdy}$.

To simplify the analysis, it is considered that the period $\Lambda$ is the same for the whole grating and the amplitude of both negative and positive coupling coefficient is the same for all periods of the grating, i.e. $\left|\kappa_{\mathrm{i}+}\right|=\left|\kappa_{\mathrm{i}-}\right|=\kappa$. This situation corresponds to the grating with a constant corrugation height $h$. It is also assumed that the propagations constants are not modified by the grating and that loading effects [10] are negligible. 


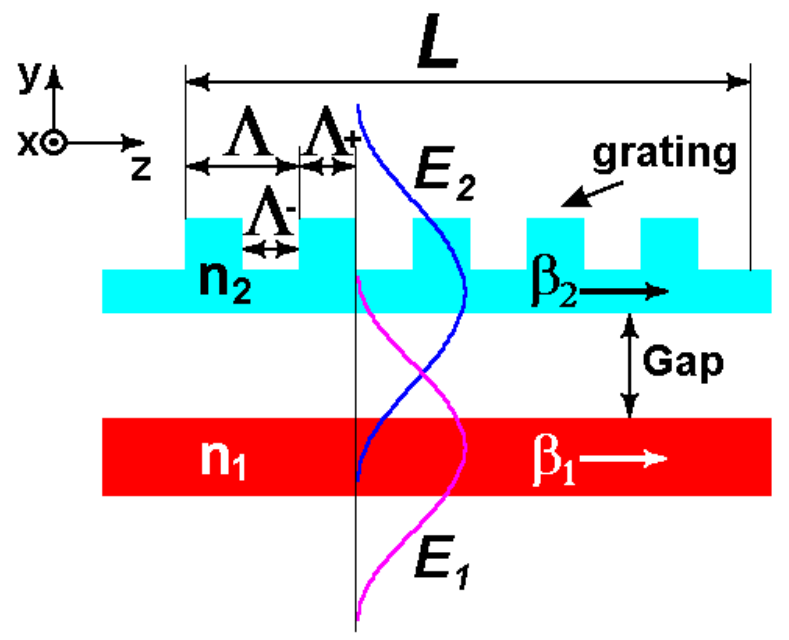

Fig. 2. Schematic of a GADC with constant corrugation height rectangular grating.

The common point of all conventional apodization schemes like the raised cosine, Gaussian or Hamming functions is the coupling strength weighting. The coupling coefficient is highest in the middle of the device and gradually decreases toward the ends of the grating. The coupling profile is usually determined by finding the average value of the coupling coefficient for each period of the grating. For a given coupling profile, the resulting apodized spectral response is then obtained using the conventional coupled mode theory (CMT) transfer matrix method $[11,12]$.

This type of approach is well suited when the coupling strength weighting is performed by varying the grating amplitude [13] or the interwaveguide separation [3,14]. However, as we will show below, it becomes inappropriate for apodization performed by duty cycle variation methods.

To demonstrate this, we consider the BDC variation method. In this case, the variation of the coupling strength along the propagation direction $z$ is obtained by changing the ratio $\rho=\Lambda_{+} / \Lambda$ between the section lengths where the coupling coefficient is positive and those where it is negative (Fig. 2). The average value $\chi_{\mathrm{i}}$ of the coupling coefficient over an $i$-th period of the grating is proportional to the intensity of the main harmonics when the grating coupling profile is decomposed into Fourier series. In the case of BDC modulation, the network period is constant and the first term of the Fourier series for the $i$-th grating period is given by a well-known expression:

$$
\chi_{i} \sim \frac{1}{\pi} \sin \left(\frac{\pi\left(\Lambda+\Delta_{i}\right)}{2 \Lambda}\right)=\frac{1}{\pi} \cos \left(\frac{\pi \varepsilon_{i}}{2}\right)
$$

Here $\varepsilon_{\mathrm{i}}=\Delta_{\mathrm{i}} / \Lambda$ is the relative change in length of the grating section. By convention, we associate $\varepsilon$ with a grating section where the coupling coefficient is positive. The modulation $\varepsilon$ is maximum at the ends of the grating, where the coupling strength is reduced, and gradually decreases toward the middle of the grating, where the coupling strength is maximum.

It is important to note that the Fourier term given by the Eq. (1) is not sensitive to the sign of $\varepsilon$. This means that the same average value coupling profile $\chi(z)$ can be obtained for different grating coupling profiles $\kappa(\mathrm{z})$. To establish the number of possible $\kappa(\mathrm{z})$ configurations, note that the variation of $\varepsilon$ from the maximum values - at the ends of the grating - to zero - in the middle of the grating - can be done in two ways. The duty cycle variation can be either an odd: $\varepsilon_{\mathrm{i}}=-\varepsilon_{-\mathrm{i}}$ or an even: $\varepsilon_{\mathrm{i}}=\varepsilon_{-\mathrm{i}}$ function with respect to the middle of the grating. In addition, for each modulation parity, the sign of the duty cycle can be reversed to its opposite $\varepsilon_{\mathrm{i}} \rightarrow-\varepsilon_{\mathrm{i}}$. This situation is illustrated in Fig. 3 with the example of $\kappa(\mathrm{z})$ coupling profiles for BDC modulated grating of length $\mathrm{L}$. 
It can be thought that there are four other possible configurations that can be obtained by performing an inverted mirror transformation of the grating shown in Fig. 3. This operation corresponds to reversing the sign of the coupling profile. However, no new configurations appear with such a transformation. Under the coupled mode theory approach (see next section), the spectral response of the grating is invariant to the sign inversion of the coupling coefficients. Thus, finally, only four configurations are possible.

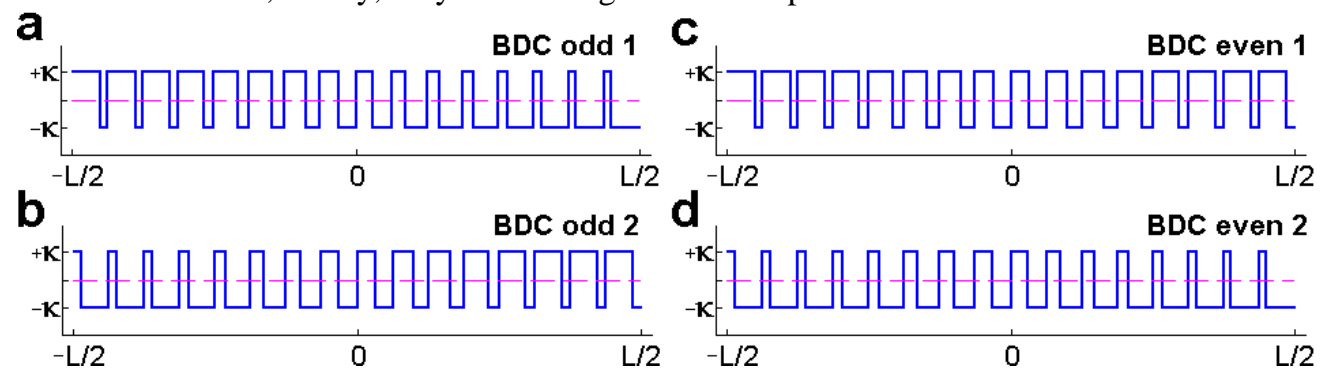

Fig. 3: BDC method modulated coupling profile: a), b) - odd $\varepsilon$ modulation; c), d) - even $\varepsilon$ modulation.

Because the average value $\chi(\mathrm{z})$ coupling profiles are the same, the conventional CMT does not make distinction between the configurations shown in Fig. 3. Therefore, the spectral responses of the four configurations are identical. To check if this is the case, we use the nonorthogonal CMT [15]. The advantage of this approach is that it allows us to analytically treat the case of a rectangular shaped grating with positive and negative coupling sections of arbitrary length without having to determine the average coupling coefficient. (The essence of this method is presented in the next section. Details on the GADC structure used for modeling are presented in Supplementary Materials).

We first consider the case of grating with an integer number of periods. Figure 4 shows the spectral responses corresponding to the grating shown in Fig. 3. While the average coupling profiles $\chi(\mathrm{z})$ are identical, it appears that the spectral responses differ and depend on the parity of the duty cycle modulation.
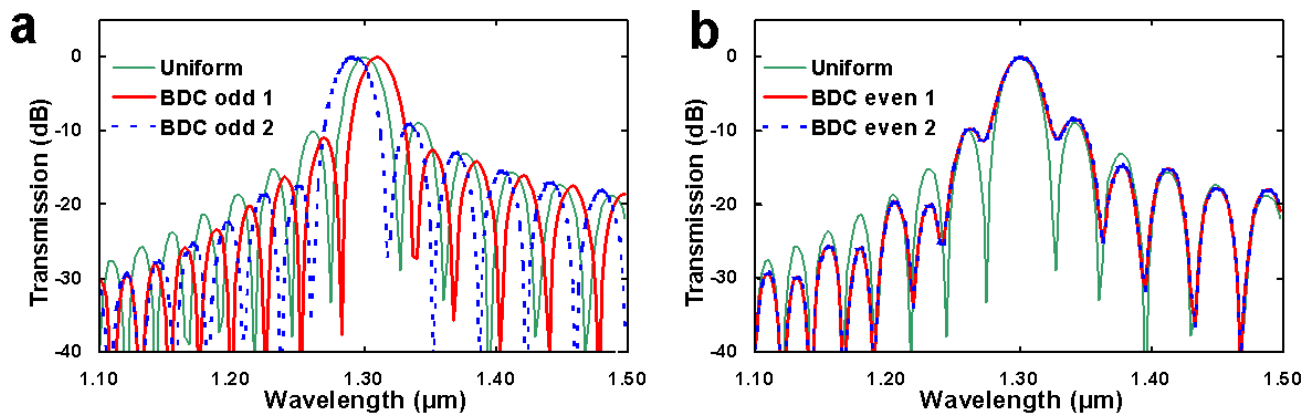

Fig. 4: Spectral responses of the BDC modulated GADC: a) Odd $\varepsilon$ modulation; b) Even $\varepsilon$ modulation. Also represented the spectral response of uniform GADC.

For the odd duty cycle modulation (Fig. 3a,b), two distinct spectral responses are observed. They are shifted in wavelength in opposite directions (Fig. 4a) from the central wavelength of the uniform grating. Thus, the central wavelength is shifted to longer wavelengths for the grating shown in Fig. 3a and to shorter wavelengths for the grating shown in Fig. 3b. An asymmetry is also visible in their respective spectral responses.

For even duty cycle modulation, as can be seen in Fig. 4b, there is no center wavelength shift. The spectral responses of the grating shown in Figs $3 \mathrm{c}$ and $3 \mathrm{~d}$ are identical. The asymmetry of the spectral response is also less significant. However, the suppression of the first sidelobe is worse than in the case of odd duty cycle modulation. 
It is important to note that only the case of even duty cycle modulation with an integer number of periods has been considered in the literature. The identical spectral response obtained in this case is probably the reason why the other symmetric duty cycle modulation schemes have been neglected so far. The same spectral response, however, is a simple consequence of the fact that the grating in Figs. $3 \mathrm{c}$ and $3 \mathrm{~d}$ are "conjugated" by the inversion symmetry relationship existing between them. Reversal symmetry corresponds to the inversion of the sign of the coupling profile with a change in light injection from left to right. The spectral response remains invariant under such an operation [16].

It can be assumed that an even modulation of the duty cycle is necessary to keep the same center wavelength. To verify this, we consider the case of a grating with an extra half-period inserted in the middle of the grating (Figs. 5a-d). The length of half-period for the inserted structure is selected to comply with the phase matching condition for the center wavelength



Fig. 5 : BDC method modulated coupling profile with additional half grating period: a), b) Odd $\varepsilon$ modulation. c), d) Even $\varepsilon$ modulation.

Although the symmetry of the duty cycle modulation is unchanged and at first glance the grating with an extra half-period are quite similar to the grating in Fig. 3, the spectral responses are completely opposite. The spectral behavior resembles that of a symmetry swap of the duty cycle modulation. This time, a center wavelength shift is observed for even duty cycle modulation (Figs 5c and 5d).

No center wavelength shift and identical spectral response are observed for the odd duty cycle modulation (Figs. 6a and 6b). The reason for the same spectral response is the mirror symmetry relationship existing between the grating in Figs. $5 \mathrm{a}$ and $5 \mathrm{~b}$. This mirror symmetry operation corresponds to a change in light injection from the left to the right side, which does not influence the spectral behavior.
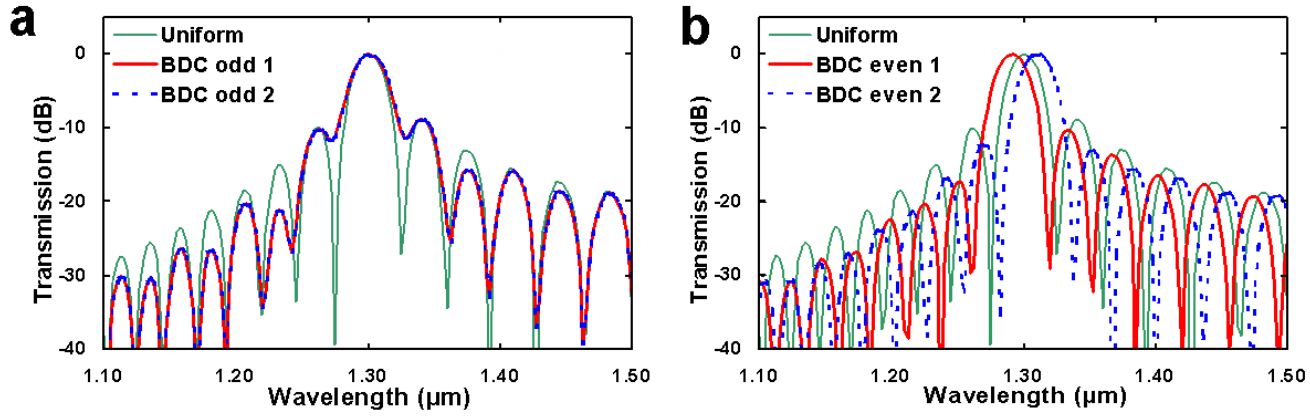

Fig. 6: Spectral responses of the BDC modulated GADC with additional half grating period: a) Odd $\varepsilon$ modulation. b) Even $\varepsilon$ modulation. Also represented the spectral response of uniform GADC.

The above examples show that the spectral behavior of cycle-modulated grating depends on the symmetry of the coupling profile. Gratings "conjugated" by inversion or mirror 
symmetry operations exhibit identical spectral response. Although these symmetry conditions appear to be essential, it is not clear whether they are mandatory to maintain the center wavelength position. The reason for the center wavelength shift observed for other configurations is also unclear. None of these effects are predicted by conventional CMT. To explain the results obtained, we have developed a perturbation approach for the BDC modulated grating transfer matrix which we present in the next section.

\section{The perturbation approach for the transfer matrix of the BDC modulated grating}

To derive the approximate expression for the transfer matrix of the BDC-modulated grating, we consider the above example of a rectangular-shaped grating directional coupler with an integer number of periods. Such a GADC is well described by a non-orthogonal coupled mode formalism developed in [15]. This approach takes into account all Fourier components of the coupling profile and is not limited to the main harmonic coupling coefficient only. For the co-directional case, the transfer matrix for sections with positive and negative coupling is respectively:

$$
\begin{aligned}
& M^{+}=\left[\begin{array}{cc}
m & -n \\
-n & m^{*}
\end{array}\right] \\
& M^{-}=\left[\begin{array}{cc}
m & n \\
n & m^{*}
\end{array}\right]
\end{aligned}
$$

For the coefficients of matrices $m$ and $n$ by adopting the same definitions as in [15], we get:

$$
\begin{aligned}
& m=\cos \left[\Omega\left(z_{i}-z_{i-1}\right)\right]-j \frac{\delta}{\Omega} \sin \left[\Omega\left(z_{i}-z_{i-1}\right)\right] \\
& n=-j \frac{\kappa}{\Omega} \sin \left[\Omega\left(z_{i}-z_{i-1}\right)\right] \\
& \text { where } \Omega=\sqrt{\delta^{2}+\kappa^{2}} \\
& \delta=\frac{\beta_{1}-\beta_{2}}{2}=\frac{\pi}{\Lambda}
\end{aligned}
$$

where $\beta_{1}$ and $\beta_{2}$ are waveguides propagation constants. In the case of a weak coupling:

$$
\Omega=\sqrt{\delta^{2}+\kappa^{2}} \approx \delta=\frac{\pi}{\Lambda}
$$

The phase matching condition for a half-period of grating is equivalent to a maximal mode intensity transfer from one waveguide to another. In this case the factors sine and cosine of elements $m$ and $n$ of the matrix $\mathrm{M}^{ \pm}$verify:
$\sin (\Omega \Lambda / 2)=1$ and $\cos (\Omega \Lambda / 2)=0$

For the BDC method, on some $i$-th grating period the length of the section with positive coupling is $\left(\Lambda+\Delta_{\mathrm{i}}\right) / 2$, while for the negative coupling section the length is $\left(\Lambda-\Delta_{\mathrm{i}}\right) / 2$. When the introduced variation is small, by using a linear approximation and by neglecting second and higher order terms in $\varepsilon_{\mathrm{i}}$ we get:

$$
\cos \left[\Omega\left(\Lambda+\Delta_{\mathrm{i}}\right) / 2\right]=\sin \left(\Omega \Delta_{\mathrm{i}} / 2\right) \approx \sin \left(\frac{\pi}{2} \frac{\Delta_{\mathrm{i}}}{\Lambda}\right) \approx \frac{\pi}{2} \varepsilon_{i}
$$

while:

$$
\sin \left[\Omega\left(\Lambda+\Delta_{\mathrm{i}}\right) / 2\right] \approx 1
$$

The coefficients $m$ and $n$ of the section transfer matrix $\mathbf{M}^{ \pm}$are then approximately:

$$
\begin{aligned}
& m \approx \pm \frac{\pi}{2} \varepsilon-j \frac{\delta}{\Omega} \\
& n \approx \mu j \frac{\kappa}{\Omega}
\end{aligned}
$$

The transfer matrix $\mathrm{M}$ of one grating period is then: 


$$
M=M^{-} M^{+}=\left[\begin{array}{cc}
\frac{\pi}{2} \varepsilon-j \frac{\delta}{\Omega} & j \frac{\kappa}{\Omega} \\
j \frac{\kappa}{\Omega} & \frac{\pi}{2} \varepsilon+j \frac{\delta}{\Omega}
\end{array}\right] \cdot\left[\begin{array}{cc}
-\frac{\pi}{2} \varepsilon-j \frac{\delta}{\Omega} & -j \frac{\kappa}{\Omega} \\
-j \frac{\kappa}{\Omega} & -\frac{\pi}{2} \varepsilon+j \frac{\delta}{\Omega}
\end{array}\right]
$$

After multiplication and by retaining only the first order terms in $\varepsilon$ :

$$
\mathrm{M} \approx\left[\begin{array}{cc}
\frac{\kappa^{2}-\delta^{2}}{\Omega^{2}} & -2 \frac{\delta \kappa}{\Omega^{2}}-2 \mathrm{j} \frac{\pi}{2} \varepsilon \frac{\kappa}{\Omega} \\
2 \frac{\delta \kappa}{\Omega^{2}}-2 \mathrm{j} \frac{\pi}{2} \varepsilon \frac{\kappa}{\Omega} & \frac{\kappa^{2}-\delta^{2}}{\Omega^{2}}
\end{array}\right]
$$

By using the same definitions as in [15]:

$$
\begin{aligned}
& \cos (\Phi)=\frac{\delta^{2}-\kappa^{2}}{\Omega^{2}} \\
& \sin (\Phi)=\frac{2 \delta \kappa}{\Omega^{2}}
\end{aligned}
$$

the following expression holds for the transfer matrix of one grating period:

$$
\mathrm{M} \approx-\left[\begin{array}{cc}
\cos (\Phi) & \sin (\Phi) \\
-\sin (\Phi) & \cos (\Phi)
\end{array}\right]-\mathrm{j} \pi \frac{\kappa}{\Omega} \varepsilon\left[\begin{array}{ll}
0 & 1 \\
1 & 0
\end{array}\right]
$$

The matrix $\mathrm{M}_{\mathrm{i}}$ is a sum of two matrices:

$\mathrm{M}_{\mathrm{i}}=\mathrm{U}+\mathrm{V}_{\mathrm{i}}$

where the matrix $U$ represents the initial grating period while the matrix $V_{i}$ takes into account the perturbation introduced by the duty cycle variation $\varepsilon_{\mathrm{i}}$ :

$$
\begin{aligned}
& U=-\left[\begin{array}{cc}
\cos (\Phi) & \sin (\Phi) \\
-\sin (\Phi) & \cos (\Phi)
\end{array}\right] \\
& \mathrm{V}_{\mathrm{i}}=\mathrm{j} \pi \frac{\kappa}{\Omega} \varepsilon_{\mathrm{i}}\left[\begin{array}{ll}
0 & 1 \\
1 & 0
\end{array}\right]
\end{aligned}
$$

The assumption made at the beginning was that all the initial grating periods $\Lambda$ are identical. However, the variation $\varepsilon_{\mathrm{i}}$ can be arbitrary for each grating period. The transfer matrix $\mathrm{M}_{\Sigma}$ for a complete coupler with $\mathrm{N}$ grating periods is then:

$$
M_{\Sigma}=\prod_{i=1}^{N}\left(U+V_{i}\right) \approx U^{N}+V_{N} U^{N-1}+U V_{N-1} U^{N-2}+\ldots+U^{N-1} V_{1}
$$

The approximation in the above expression comes from the fact that after evaluation of the product, terms higher than first order in $\varepsilon_{\mathrm{i}}$ are neglected. To calculate the product $\mathrm{V}_{\mathrm{i}} \mathrm{U}^{\mathrm{i}-1}$ it is easy to see that:

$$
U^{i-1}=(-1)^{i-1}\left[\begin{array}{cc}
\cos (\Phi) & \sin (\Phi) \\
-\sin (\Phi) & \cos (\Phi)
\end{array}\right]^{i-1}=(-1)^{i-1}\left[\begin{array}{cc}
\cos [(i-1) \Phi] & \sin [(i-1) \Phi] \\
-\sin [(i-1) \Phi] & \cos [(i-1) \Phi]
\end{array}\right]
$$

So after multiplication the approximate expression for the grating assisted co-directional coupler transfer matrix modulated by the BDC method is:

$$
\mathrm{M}_{\Sigma}=(-1)^{\mathrm{N}}\left[\begin{array}{cc}
\cos (\mathrm{N} \Phi) & \sin (\mathrm{N} \Phi) \\
-\sin (\mathrm{N} \Phi) & \cos (\mathrm{N} \Phi)
\end{array}\right]+\mathrm{j}(-1)^{\mathrm{N}} \pi \frac{\kappa}{\Omega} \sum_{-(N-1) / 2}^{(N-1) / 2} \varepsilon_{\mathrm{i}}\left[\begin{array}{cc}
-\sin (2 i \Phi) & \cos (2 i \Phi) \\
\cos (2 i \Phi) & \sin (2 i \Phi)
\end{array}\right]
$$

The first matrix corresponds to the initial unmodulated grating while the second represents the perturbation introduced by the modulation. This approximate expression of the transfer matrix can now be used to explain the spectral behavior of the BDC modulated grating.

Thus, to understand the origin of this difference between even and odd duty cycle modulations, let us take a closer look at Eq. (23). 
For the unmodulated uniform grating, a full-mode intensity transfer occurs at the central wavelength $\lambda_{0}$, satisfying the phase matching condition $N \Phi=\pi / 2$. The main diagonal terms cancel while the cross terms are \pm 1 .

The situation is different in the presence of grating modulation. The diagonal terms of the perturbation matrix [second term on right side of Eq. (23)] modify the phase matching condition and produce a shift in the center wavelength that depends on the sign of the modulation of $\varepsilon_{\mathrm{i}}$. The cross terms in the perturbation matrix produce additional mode coupling. This results in a higher sidelobe level in the vicinity of the center wavelength.

Thus, in the case of even duty cycle modulation, only the main diagonal terms of the perturbation matrix cancel:

$$
\sum_{-(N-1) / 2}^{(N-1) / 2} \varepsilon_{i} \sin (2 i \Phi)=0
$$

This explains the lack of offset for the center wavelength. The non-canceling cross terms of the perturbation matrix are responsible for the higher level of the first sidelobe.

For the odd duty cycle modulation shown in Figs. $3 a$ and $3 b$, the center wavelength offset is explained by the fact that only the cross terms of the perturbation matrix cancel:

$$
\sum_{-(N-1) / 2}^{(N-1) / 2} \varepsilon_{i} \cos (2 i \Phi)=0
$$

but not the main diagonal terms.

The derivation of the transfer matrix expression with an additional half period of grating in the middle of the structure is the same as in the case of an integer number of periods. By straightforward calculations, the expression for the perturbation matrix is then:

$$
\mathrm{j}(-1)^{\mathrm{N}} \pi \frac{\delta}{\Omega} \sum_{-(N-1) / 2}^{(N-1) / 2} \varepsilon_{\mathrm{i}}\left[\begin{array}{cc}
\cos (2 i \Phi) & \sin (2 i \Phi) \\
-\sin (2 i \Phi) & \cos (2 i \Phi)
\end{array}\right]+\mathrm{j}(-1)^{\mathrm{N}} \pi \frac{\kappa}{\Omega} \sum_{-(N-1) / 2}^{(N-1) / 2} \varepsilon_{\mathrm{i}}\left[\begin{array}{cc}
-\sin (2 i \Phi) & \cos (2 i \Phi) \\
\cos (2 i \Phi) & \sin (2 i \Phi)
\end{array}\right]
$$

The perturbation matrix is now the sum of two matrices. However, in the weak coupling approximation when $\kappa<<\delta$, only the left perturbation matrix can be retained.

For odd duty cycle modulation the diagonal terms of the left matrix in Eq. (26) cancel. This explains the absence of a shift in the center wavelength. In the case of even duty cycle modulation, the diagonal terms are responsible for the shift of the central wavelength which depends on the sign of the variation of $\varepsilon_{\mathrm{i}}$.

The analysis performed shows that despite a number of rough approximations used to develop the perturbation approach, it provides a good understanding of the spectral behavior of BDC modulated gratings. However, the most important advantage of this approach is that it also provides tools to improve and optimize the performance of the duty cycle modulated grating, as demonstrated in the next section.

\section{Supersymmetric duty cycle modulation method}

From the above analysis, it is clear that effective duty cycle modulation requires cancellation of the perturbation matrix. The BDC method fails to simultaneously cancel the cross and diagonal terms of the perturbation matrix. The apodization of the spectral response by this method is therefore not optimal.

The cancellation of the perturbation matrix is equivalent to satisfying Eqs. (24) and (25) simultaneously. Although this cannot be achieved in a strict mathematical sense, Eqs. (24) and (25) can be approximately satisfied if $\varepsilon_{\mathrm{i}}$ changes sign in an oscillatory manner:

$\varepsilon_{\mathrm{i}-1} \approx-\varepsilon_{\mathrm{i}} \approx \varepsilon_{\mathrm{i}+1} \approx-\varepsilon_{\mathrm{i}+2}$

When such an oscillating $\varepsilon_{\mathrm{i}}$ behavior is applied to the BDC method (Fig. 7), the negative coupling section length for the the $i$-1 grating is:

$\left(\Lambda-\Delta_{\mathrm{i}-1}\right) / 2$

For the $i$-th period the positive coupling section length become:

$\left(\Lambda+\left(-\Delta_{\mathrm{i}}\right)\right) / 2$ 
and the negative coupling section length become:

$\left(\Lambda-\left(-\Delta_{\mathrm{i}}\right)\right) / 2$

For the $i+1$ period the negative coupling section length is:

$\left(\Lambda+\Delta_{i+1}\right) / 2$

When $\mathrm{N}$, the number of grating is important $(\mathrm{N}>>1)$, the duty cycle variation between adjacent grating periods is relatively small and $\left|\Delta_{\mathrm{i}-1}\right| \approx\left|\Delta_{\mathrm{i}}\right| \approx\left|\Delta_{\mathrm{i}+1}\right|$. In this case it can be considered that Eqs.(28) and (29) describe a period of length $\left(\Lambda-\Delta_{\mathrm{i}}\right)$ while Eqs. (30) and (31) a period of length $\left(\Lambda+\Delta_{\mathrm{i}}\right)$. The grating with such an oscillating modulation (Fig. 7), becomes very similar to the grating modulated by the off-resonance method (Fig. 1b).

Oscillatory modulation of $\varepsilon_{\mathrm{i}}$ transforms the BDC method into a variant of the ORM [17]. In the following, we refer to this modulation scheme as the supersymmetric duty cycle (SDC) method. It was initially introduced for Parity-Time symmetric Bragg gratings to preserve the necessary quadrature phase difference between the real and imaginary parts of the complex index profile [18]. We show here that the use of this method for apodization purposes is more general and not only limited to PT-symmetric Bragg gratings.

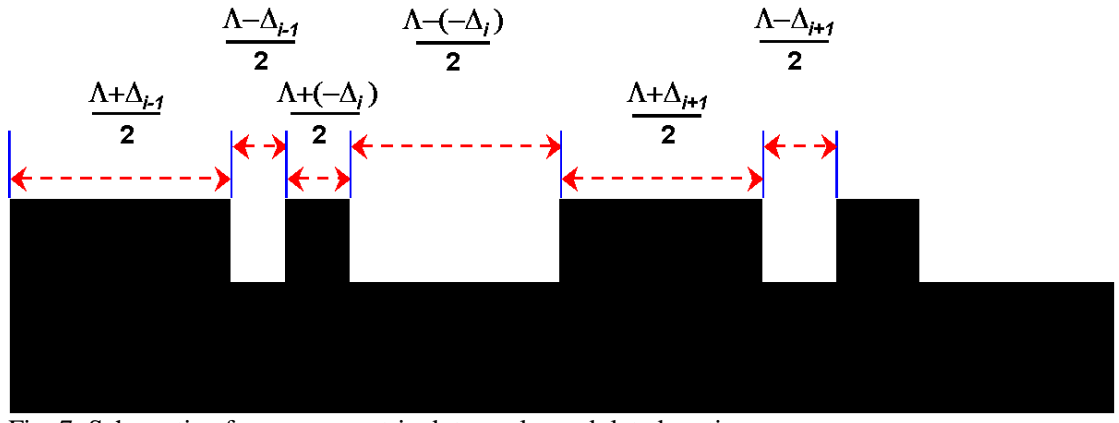

Fig. 7: Schematic of supersymmetric duty cycle modulated grating

The different configurations corresponding to the SDC method are represented in Fig. 8.

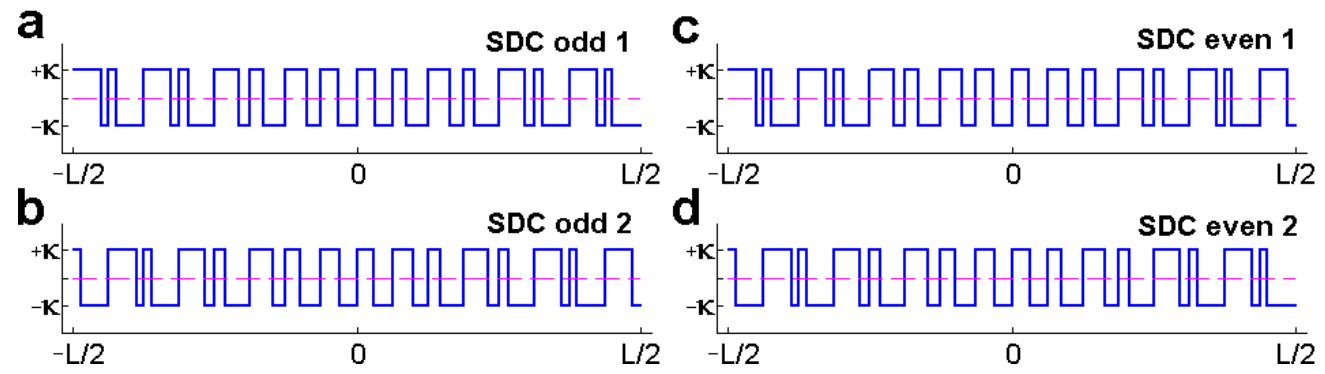

Fig. 8: SDC method modulated coupling profile: a), b) Odd $\varepsilon$ modulation. c), d) Even $\varepsilon$ modulation.

The spectral responses of the gratings modulated by the SDC method described above are shown in Fig. 9. This time, the center wavelength hardly changes regardless of the symmetry of the duty cycle modulation. The side lobe suppression is also improved. The side-lobe suppression performance of the SDC-modulated grating under even duty cycle modulation can be further improved without shifting the center wavelength by increasing the modulation ratio, which, for the purpose of fair comparison with the concatenated grating method, was limited to only $50 \%$ in the selected examples.

Furthermore, the insertion of an extra half-period into the gratings modulated by the SDC method is having no influence on the position of the center wavelength and only very little influence on the level of secondary sidelobes. The results obtained are essentially consistent with the predictions of the perturbation matrix analysis. 

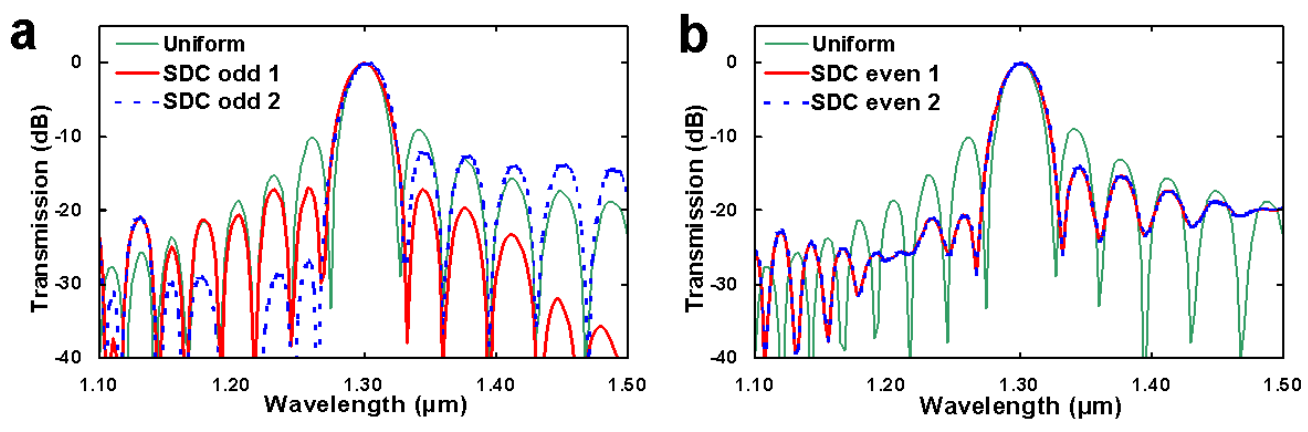

Fig. 9: Spectral responses of the SDC modulated GADC: a) Odd $\varepsilon$ modulation. b) Even $\varepsilon$ modulation. Also represented the spectral response of uniform GADC.

\section{Comparative of duty cycle variations methods}

The cancellation of the perturbation matrix is not the only reason for the better performance of the SDC and similar methods. The better suppression of sidelobes is also due to a more efficient modulation of the coupling strength produced by the SDC method. Recall that the coupling strength coefficient is proportional to the strength of the principal harmonics when the coupling profile of the grating is decomposed into Fourier series. For the SDC method, the average coupling coefficient can be obtained by considering two consecutive periods of the grating (Fig. 7). The average coupling coefficient is then:

$$
\chi_{S D C}=\frac{\sqrt{2}}{\pi} \cos ^{2}\left(\frac{\pi \Delta}{2 \Lambda}\right)
$$

For comparison the average coupling coefficient for the BDC method is:

$$
\chi_{B D C}=\frac{\sqrt{2}}{\pi} \cos \left(\frac{\pi \Delta}{2 \Lambda}\right)
$$

The average coupling coefficient is the same for the PSPG method while for the CGM it is

$$
\chi_{C G M}=\frac{\sqrt{2}}{\pi}\left|\cos \left(\frac{\pi \Delta}{\Lambda}\right)\right|
$$

The variation of the coupling strength as a function of $|\Delta| / \Lambda$, described by Eqs. (32-34) is shown in Fig. 10. As can be seen, for the same $\Delta$ modulation, the SDC produces a larger variation in coupling strength compared to the BDC. Therefore, SDC provides better apodization of the spectral response compared to the BDC method.

An even larger variation in the average coupling strength is obtained for the concatenated gratings method (Fig. 1d). However, the main drawback of this CGM is that it requires, especially for low modulation, very short periods of length $\Delta$, which is a serious obstacle in terms of practical realization. Therefore, in practice, the use of the SDC method or a similar method is more affordable in terms of manufacturing technology requirements. Additional details on apodization using PSPG and CGM are provided in Supplementary Materials. 


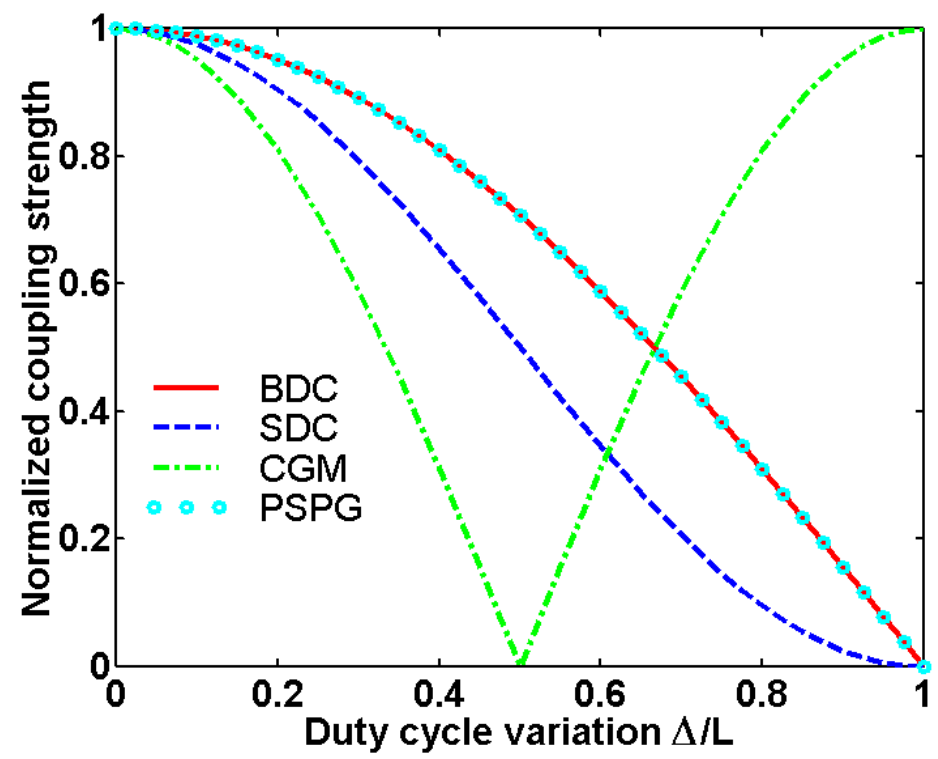

Fig. 10: Variation of the average coupling strength for different duty cycle modulation methods.

\section{Conclusion}

The overview of the different grating duty cycle modulation methods shows that the SDC and similar methods performs better than the others. Its advantage is that it does not change the position of the center wavelength and achieves better suppression of the sidelobes. The main principle of this method is the oscillatory variation of the grating duty cycle with an oscillation amplitude that increases towards the extremities and decreases at the center of the grating. The essence of the SDC method can be understood on the basis of the perturbation matrix analysis. For conventional methods, such as BDC, through an appropriate choice of grating symmetry (mirror or inversion), it is possible to cancel either the diagonal terms responsible for the center wavelength shift or the cross terms responsible for the sidelobe level, but not both simultaneously. In the SDC method, the oscillatory variation of the grating duty cycle allows the diagonal and cross terms of the perturbation matrix to be cancelled approximately simultaneously.

The analysis of the perturbation matrix allows thus to understand the disadvantages that are inherent to other methods of modulating the grating duty cycle. In addition, the analysis of the perturbation matrix allows us to find suitable solutions to remedy the situation.

\section{Disclosures}

The authors declare no conflicts of interest.

\section{References}

1. H. A.Haus, W-.P. Huang, S. Kawakami, N.A. Whitaker, "Coupled-mode theory of optical waveguides," J. Lightwave Technol. LT-5(1), 16-23 (1987).

2. H. Kogelnik, "Filter response of non-uniform almost-periodic structures," Bell System Technical Journal 55(1), 109-126 (1976).

3. R.C. Alferness, P.S. Cross, "Filter characteristics of codirectionally coupled waveguides with weighted coupling,” IEEE J. Quantum. Electron. QE-14(11), 843-847 (1978).

4. J.Albert, K.O. Hill, B. Malo, S. Thériault, F. Bilodeau, D.C. Jonson, L.E. Erickson, "Apodisation of the spectral response of fibre Bragg gratings using a phase mask with variable difraction efficiency," Electronic Lett. 31(3), 222-223 (1995). 
5. H. Sakata, "Sidelobe suppression in grating-assisted wavelength-selective couplers," Opt. Lett. 17(7), 463-465 (1992).

6. D.-B. Kim, C.-Y. Park, B.-H. O, H.-M. Kim, T.-H. Yoon, "Fabrication of sidelobe-suppressed InPInGaAsP vertical-coupler optical filter using pair grating structure," IEEE Photonic Technology Letters, 10(11), 1593-1595 (1998).

7. A. Lupu, A. Carenco, P. Win, H. Sik, P. Boulet, M. Carré, S. Slempkes, "Spectral Response Apodization of Bragg-Like Optical Filters with a Novel Grating Chirp Design," In OFC/IOOC. Technical Digest. Optical Fiber Communication Conference, 1999, and the International Conference on Integrated Optics and Optical Fiber Communication (2, 271-273). IEEE.

8. D. Wiesmann, D, Erni, H. Rothuizen, R. Germann, G.-L. Bonna, C. David, H. Jäckel, "Apodization of a grating filter by concatenation of a Bragg gratings with different ridge patterns," Proc. ECIO'99, 159-162.

9. D. Wiesmann, C. David, R. Germann, D. Erni, G.L. Bonna, "Apodized surface-corrugated gratings with varying duty cycles," IEEE Photonic Technology Letters 12(6), 639-641 (2000).

10. B. Little, "Junction problem and loading effects in grating-assisted couplers," Opt. Lett. 21(13), 949-951 (1996).

11. W.-P. Huang, H.A. Haus, "Power exchange in grating assisted couplers," J. Lightwave Technol. 7(6), 920-924 (1989).

12. W.-P. Huang, "Coupled-mode theory for optical waveguides: an overview," J. Opt. Soc. Am. A 11(3), 963-983 (1994).

13. P.S. Cross, H. Kogelnik, "Sidelobe suppresson in corrugated-waveguide filters," Opt. Lett. 1(1), 43-45 (1977).

14. R.C. Alferness, "Optical directional couplers with weighted coupling," Appl. Phys. Lett., 35(3), 260-262 (1979).

15. W.-P. Huang, J.W.Y. Lit, "Nonorthogonal coupled-mode theory of grating-assisted couplers," J. Lightwave Technol. 9(7), 845-852 (1991).

16. G.H. Song, "Theory of symmetry in optical filter response," J. Opt. Soc. Am. 11(7), 2027-2037 (1994)

17. In the initial ORM the sections with positive and negative coupling are exactly of the same length. Despite some symmetry differences in the coupling profiles, the performances of the two methods are quite similar, therefore only SDC results are presented in the actual paper.

18. A. Lupu, H. Benisty, A. Lavrinenko, "Tailoring spectral properties of binary PT-symmetric gratings by using duty cycle methods," JSTQE 22(5), 35-41 (2016). 